\title{
THE OPERATION OF AN ISFET AS AN ELECTRONIC DEVICE
}

\section{P BERGVELD}

Afdeling der Electrotechniek, Technische Hogeschool Twente, Enschede, POB 217 (The Netherlands)

\section{Introduction}

The conventional ion sensitive sensors, such as glass membrane electrodes, coated wire electrodes, metal-metal oxıde electrodes, etc, convert a non-electrical quantity into an electrical quantity, which can then be measured with appropriate electronic circuitry connected in series Unpredictable deviations in transducer parameters cannot be controlled in such a measurnng system Because the ISFET also has an electrical input besides the 1on-sensitive input, this results in unique application possibilities, with consequences for the development of adequate electronic circuitry In order to obtain an insight into this matter, it is useful first to understand the electrical behaviour of a MOSFET, from which the ISFET behaviour can be denved. Based on this theory some electronic design starting points can be developed for an appropriate ISFET application

\section{The MOSFET and ISFET small-sıgnal behaviour}

In first-order MOS transistor theory, the equation for the drain current, $I_{\mathrm{d}}$, in the unsaturated region $\left(V_{\mathrm{d}}<V_{\mathrm{g}}-V_{\mathrm{t}}\right)$ is

$$
I_{\mathrm{d}}=\beta\left[\left(V_{\mathrm{g}}-V_{\mathrm{t}}\right) V_{\mathrm{d}}-\frac{1}{2} V_{\mathrm{d}}^{2}\right]
$$

where $\beta=\mu C_{\mathrm{ox}} W / L$ is a geometry constant, $V_{\mathrm{g}}$ and $V_{\mathrm{d}}$ are the $\mathrm{d} \mathrm{c}$ gate to source and drain to source voltages, respectively, and $V_{t}$ is the threshold voltage which represents all the effects of substrate depletion charge, work function of gate metal, interface states, and fixed charges in the oxide

The equation for the a $c$ drain current, $\imath_{d}$, is given by

$$
l_{\mathrm{d}}=S v_{\mathrm{g}}+\frac{1}{R_{\mathrm{ch}}} v_{\mathrm{d}}
$$

where $v_{\mathrm{g}}$ and $v_{\mathrm{d}}$ are the a c. gate to source and drain to source voltages, respectively, $S$ is the mutual conductance and $R_{\mathrm{ch}}$ is the differential channel resistance Expressions for $S$ and $R_{\mathrm{ch}}$ follow from differentiations

$$
S=\left.\frac{\mathrm{d} I_{\mathrm{d}}}{\mathrm{d} V_{\mathrm{g}}}\right|_{V_{\mathrm{d}}=\text { const }}=\beta V_{\mathrm{d}}
$$




$$
\frac{1}{R_{\mathrm{ch}}}=\left.\frac{\mathrm{d} I_{\mathrm{d}}}{\mathrm{d} V_{\mathrm{d}}}\right|_{V_{\mathrm{g}}=\mathrm{const}}=\beta\left[V_{\mathrm{g}}-V_{\mathrm{t}}-V_{\mathrm{d}}\right]
$$

The question arises whether these equations also hold for ISFETs or whether they must be modified For an ISFET, $V_{\mathrm{g}}$ is kept constant and $V_{\mathrm{t}}$ contains the variable input signal, while in the case of a MOSFET, $V_{t}$ is assumed to be constant and $V_{\mathrm{g}}$ is the variable

Seen electronically, this difference in basis conception will give no complications, because the term $V_{\mathrm{g}}-V_{\mathrm{t}}$ can be seen in both cases as the actual input variable

Considering further the difference between a MOSFET and an ISFET, besides the change in input variable, the method of contacting the actual source and drain is also necessarily quite different With a MOSFET the source and drain regions can, in principle, be completely evaporated with aluminum, which makes a very low resistive contact after alloying By contrast, with ISFETs this contact method is impossible because the gate has to be contacted by an electrolyte, which means that no metal contacts can exist in the vicinity of this area Depending on the necessary length of the insulating lacquers over the oxidized source and drain regions, the contact places are usually some millimeters away from the actual source- and drainto-gate interfaces The most common ISFET configuration is shown in Fig 1

Using a donor concentration $N_{\mathrm{D}}=5 \times 10^{19} \mathrm{~cm}^{-3}$ for the source and drain regions, resulting in a square resistance $R=40 \Omega$, internal source and drain resistances are created with a value $40 \times l / w \Omega$, in which $l / w$ is the length/width ratio of the diffusion region In the case of the geometry as shown in Fig 1, the series resistance of source and drain will thus be $4 \times$ $40 \Omega=160 \Omega$ In practical cases of needle-shaped ISFETs, this value is even larger (Esashi et al, 1978) This internal resistance of source and drain, of which the value depends, of course, on the actual geometry of the device, will never be zero, and gives nise to serious problems with regard to the device parameters as derived in eqns (3) and (4) This can easily be seen from the following calculations, based on the model given in Fig 2

$$
\begin{aligned}
& V_{\mathrm{d}}=V_{\mathrm{d}^{\prime} \mathrm{s}^{\prime}}+I_{\mathrm{d}}\left(R_{\mathrm{s}}+R_{\mathrm{d}}\right) \\
& V_{\mathrm{g}}=V_{\mathrm{gs}^{\prime}}+I_{\mathrm{d}} R_{\mathrm{s}}=V_{\mathrm{gs}^{\prime}}+\frac{V_{\mathrm{d}}-V_{\mathrm{d}^{\prime} \mathrm{s}^{\prime}}}{2}\left(\text { for } R_{\mathrm{d}}=R_{\mathrm{s}}\right)
\end{aligned}
$$

Substituting for $V_{\mathrm{g}}$ and $V_{\mathrm{d}}$ of eqn (1), $V_{\mathrm{gs}}$ (eqn (6)) and $V_{\mathrm{d}^{\prime} \mathrm{s}^{\prime}}$ (eqn (5)) respectively, gives

$$
\begin{aligned}
I_{\mathrm{d}} & =\beta\left[\left(V_{\mathrm{g}}-\frac{1}{2} V_{\mathrm{d}}+\frac{1}{2} V_{\mathrm{d}^{\prime} \mathrm{s}^{\prime}}-V_{\mathrm{t}}\right) V_{\mathrm{d}^{\prime} \mathrm{s}^{\prime}}-\frac{1}{2} V_{\mathrm{d}^{\prime} \mathrm{s}^{\prime}}{ }^{2}\right] \\
& =\beta\left[\left(V_{\mathrm{g}}-\frac{1}{2} V_{\mathrm{d}}-V_{\mathrm{t}}\right)\left(V_{\mathrm{d}}-I_{\mathrm{d}}\left(R_{\mathrm{d}}+R_{\mathrm{s}}\right)\right)\right]
\end{aligned}
$$

From eqn (7) the equation can be derived for the drain current of a transistor with internal resistances

$$
I_{\mathrm{d}}=\beta V_{\mathrm{d}} \frac{V_{\mathrm{g}}-\frac{1}{2} V_{\mathrm{d}}-V_{\mathrm{t}}}{1+\beta\left(R_{\mathrm{d}}+R_{\mathrm{s}}\right)\left(V_{\mathrm{g}}-\frac{1}{2} V_{\mathrm{d}}-V_{\mathrm{t}}\right)}
$$



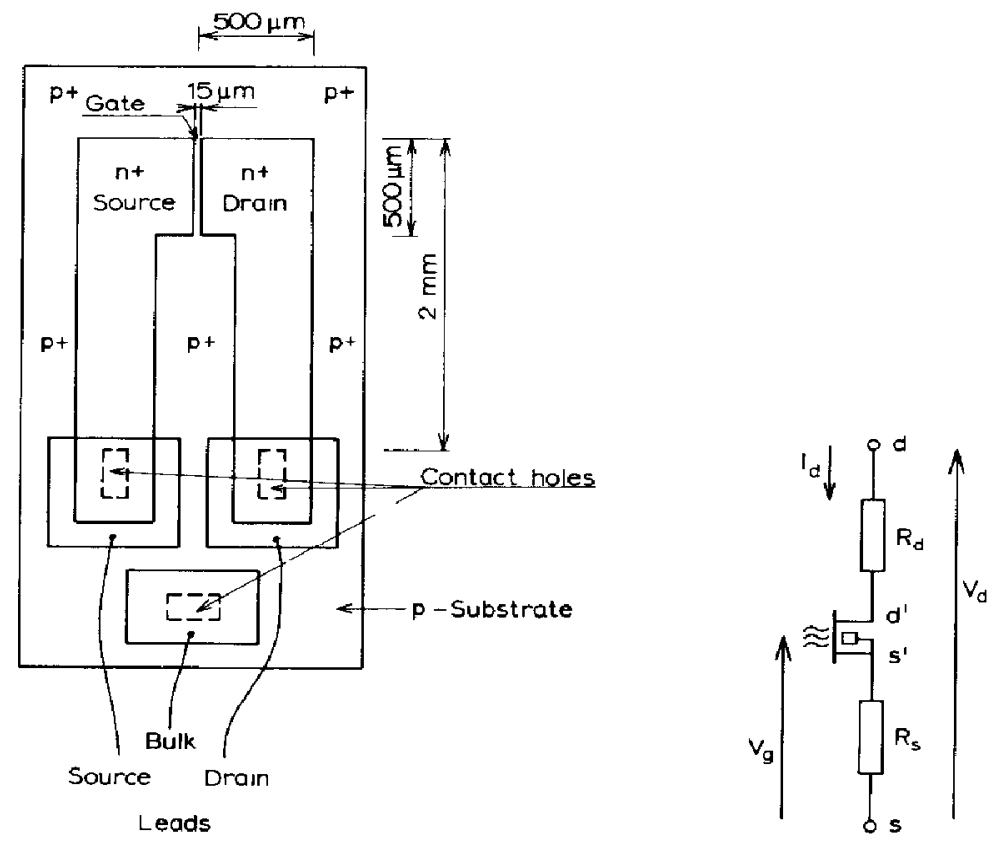

Fig 1 Geometric representation of a common ISFET configuration

Fig 2 Electronic model of an actual ISFET design with internal resistances $R_{\mathrm{s}}$ and $R_{\mathrm{d}}$

The expressions for the mutual conductance as well as the differential channel resistance, follow from differentiations

$$
\begin{aligned}
S=\left.\frac{\mathrm{d} I_{\mathrm{d}}}{\mathrm{d} V_{\mathrm{g}}}\right|_{V_{\mathrm{d}}=\text { const }}= & \beta V_{\mathrm{d}} \frac{1}{\left[1+\beta\left(R_{\mathrm{d}}+R_{\mathrm{s}}\right)\left(V_{\mathrm{g}}-\frac{1}{2} V_{\mathrm{d}}-V_{\mathrm{t}}\right)\right]^{2}} \\
\frac{1}{R_{\mathrm{ch}}}=\left.\frac{\mathrm{d} I_{\mathrm{d}}}{\mathrm{d} V_{\mathrm{d}}}\right|_{V_{\mathrm{g}=\text { const }}}= & \frac{\beta\left(V_{\mathrm{g}}-\frac{1}{2} V_{\mathrm{d}}-V_{\mathrm{t}}\right)}{1+\beta\left(R_{\mathrm{d}}+R_{\mathrm{s}}\right)\left(V_{\mathrm{g}}-\frac{1}{2} V_{\mathrm{d}}-V_{\mathrm{t}}\right)}- \\
& -\frac{\frac{1}{2} \beta V_{\mathrm{d}}}{\left[1+\beta\left(R_{\mathrm{d}}+R_{\mathrm{s}}\right)\left(V_{\mathrm{g}}-\frac{1}{2} V_{\mathrm{d}}-V_{\mathrm{t}}\right)\right]^{2}}
\end{aligned}
$$

For $R_{\mathrm{s}}=R_{\mathrm{d}}=0$, eqns (9) and (10) change into eqns (3) and (4) The influence of $R_{\mathrm{d}}$ and $R_{\mathrm{s}}$ on the channel resistance gives no electronic problems, because it only increases $R_{\mathrm{ch}}$ A decrease of $1 / R_{\mathrm{ch}}$ decreases the influence of $v_{\mathrm{d}}$ on $l_{\mathrm{d}}$ (see eqn (2)), which is an advantage Problems, however, do arise from the influence of $R_{\mathrm{s}}$ and $R_{\mathrm{d}}$ on the mutual conductance or, in other words, the sensitivity of the device, which decreases drastically for real values of $R_{\mathrm{s}}$ and $R_{\mathrm{d}}$ This effect is shown in Fig 3 for a MOSFET with additional $R_{s}$ and $R_{d}$ In accordance with eqn (9) the sensitivity decreases more for larger values of $\left(V_{\mathrm{g}}-V_{\mathrm{t}}\right)$, as can also be seen in Fig 3

The shape of the $I_{\mathrm{d}}-V_{\mathrm{g}}$ curves published from ISFETs [1 - 3] can be fully explained as being the result of the internal source and drain resistances, the effect of which is very pronounced, especially for small values of $V_{\mathrm{d}}$ as shown in Fig 4, and in accordance with eqn (9) 
1 MOST without $R_{s}$ or $R_{d}$

2 MOST with $R_{s}=120 \Omega$ or $R_{d}=120 \Omega$

3 MOST with $R_{s}=R_{d=120 \Omega}$

$V_{\mathrm{ds}}=100 \mathrm{mV}$

$\beta=410^{3} \mathrm{AV}^{2}$

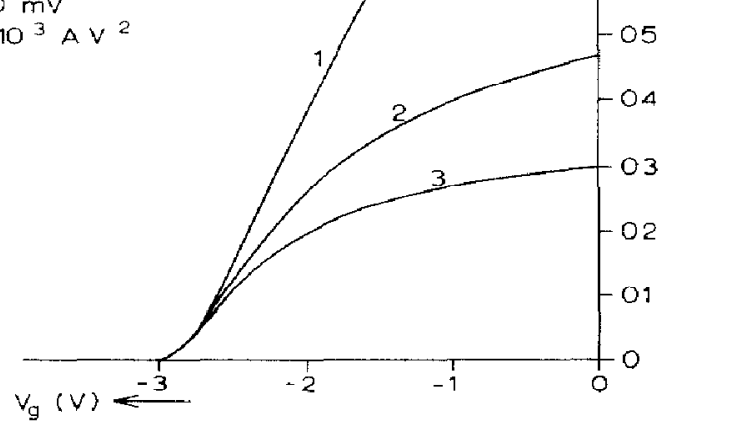

Fig $3 I_{\mathrm{d}}-V_{\mathrm{g}}$ characteristics of a MOSFET for various values of series resistors in source and drain leads, $R_{\mathrm{s}}$ and $R_{\mathrm{d}}$, respectively 1 MOSFET without $R_{\mathrm{s}}$ or $R_{\mathrm{d}}, 2$ MOSFET wath $R_{\mathrm{s}}=120 \Omega$ or $R_{\mathrm{d}}=120 \Omega, 3$ MOSFET with $R_{\mathrm{s}}=R_{\mathrm{d}}=120 \Omega, V_{\mathrm{ds}}=100 \mathrm{mV}, \beta=4 \times$ $10^{-3} \mathrm{~A} \mathrm{~V}^{-2}$

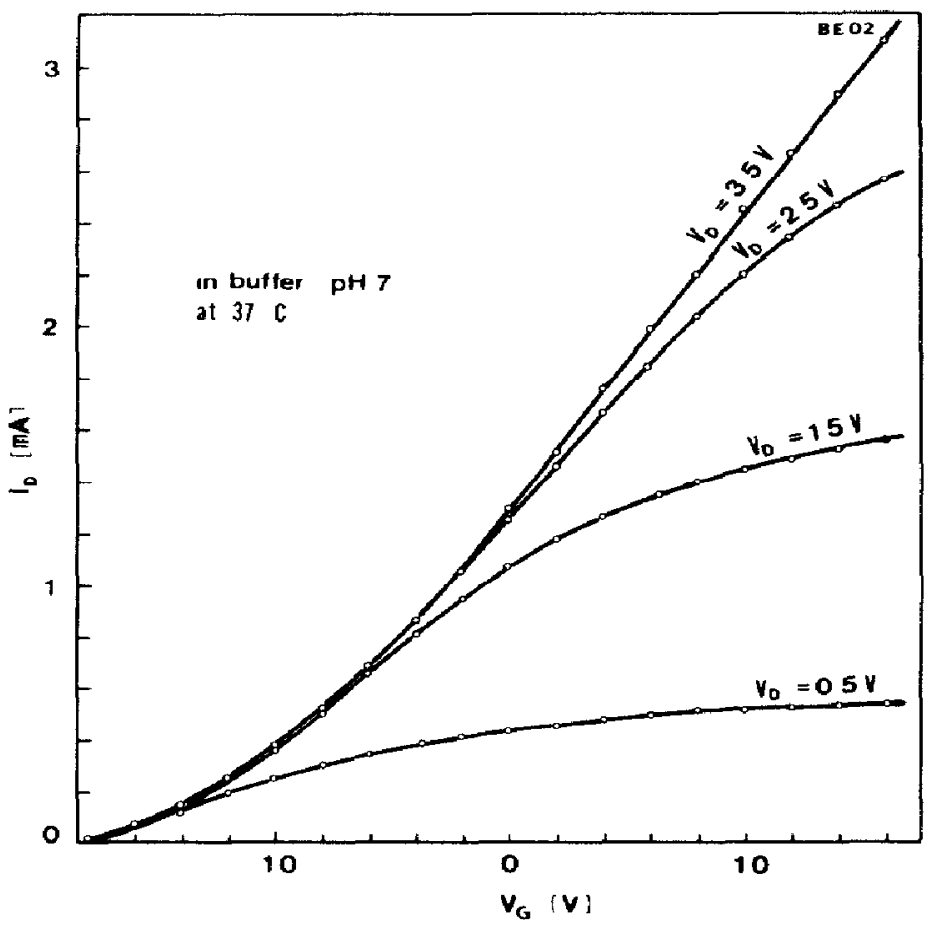

Fig $4 I_{\mathrm{d}}-V_{\mathrm{g}}$ curves of a pH ISFET (after Moss et al [2])

It can be concluded that, electronically seen, a MOSFET is a device in which the sensitivity depends only on the device parameter, $\beta$, and the applied voltage, $V_{\mathrm{d}}$ (see eqn (3)), while an ISFET is a device in which the sensitivity is dependent, in addition, on the input signal $V_{t}=f(\mathrm{pH}+\Delta \mathrm{pH})$ and its bias $V_{\mathrm{g}}$ (see eqn (9)) There are, in principle, two ways of solving this problem, namely, technologically and electronically A technological way of 
approaching the problem is to shorten the length of the source and drain diffusions by contacting the source and drain from the liquid-free side of the device However, this implies new technologies because this technique is very unusual for standard transistor devices The most promising solution is described by Cline et al [4], whlle Zemel [5] shows that it can be used for gated diodes It is, however, much easier to solve the problem of decreased sensitivity by means of an electronic circuit, which is insensitive to series resistors due to the application of the feedback principle

\section{Electronic circuit design adapted to ISFETs}

From the curves of Fig 4 it can be seen that biasing an ISFET at a constant $I_{\mathrm{d}}$, at the same time maintaining a constant $V_{\mathrm{d}}$, can only be obtained by (automatic) control of $V_{\mathrm{g}}$, compensating for a change in $\mathrm{pH}$ This control action is mentioned in the previous Section by the proposed feedback system This means, with regard to the small signal condition (eqn (2)), that $v_{d}$ is kept zero, makng the measurement insensitive to the value of $\boldsymbol{R}_{\mathrm{ch}}$, while $l_{\mathrm{d}}$ is also kept at zero by effectively controllung $v_{\mathrm{g}}$ to zero, which makes the measurement independent of $S$ and thus of $R_{\mathrm{d}}$ and $R_{s}$ The required condition can be obtained in two ways The furst possibility is control of $l_{\mathrm{d}}=0 \mathrm{in}$ a feedback loop due to automatic adjustment of the potential of the reference electrode, and thus of the liquid, in respect of the source and drain potentials The second possibility is an automatic adjustment of the source and drain potentials with regard to a constant liquid potential, usually the ground potential An example of the first possibility is the automatically balanced bridge circuit as shown in Fig 5

A change in $V_{\mathrm{t}}$ due to a $\mathrm{pH}$ variation is compensated by a change in $V_{\mathrm{g}}$ $v$ ta the reference electrode $A$ disadvantage of this system is that the liquid may not be grounded, which is sometimes required for the benefit of certain measuring conditions Also, the possibility of accidental grounding has to be prevented This problem can be solved by the use of isolation amplifiers and an isolated power supply A further disadvantage of this bridge circuit is the asymmetrical impedance of the source and drain leads, makang the system, also under floating conditions, sensitive to interference from external electric fields and static electricity

An example of the second circuit design, as mentioned above, is the source and drain follower concept, which is shown in Fig 6 In contrast to the bridge circuit shown in Fig 5, the hquid is now connected to the ground of the crrcuit by means of the reference electrode The system consists essentially of a power supply (current source and adjustable reference voltage, $V_{\text {ref }}$ ), an instrumentation amplifier system, and an operational amplifier The ISFET is connected to the leads of the instrumentation amplifier which are normally used to connect a resistor that determines the amplification of the amplifier The usual inputs of the amplifier are, in this case, connected to a fixed voltage $I R_{1}$, provided by the current source The output voltage of the 


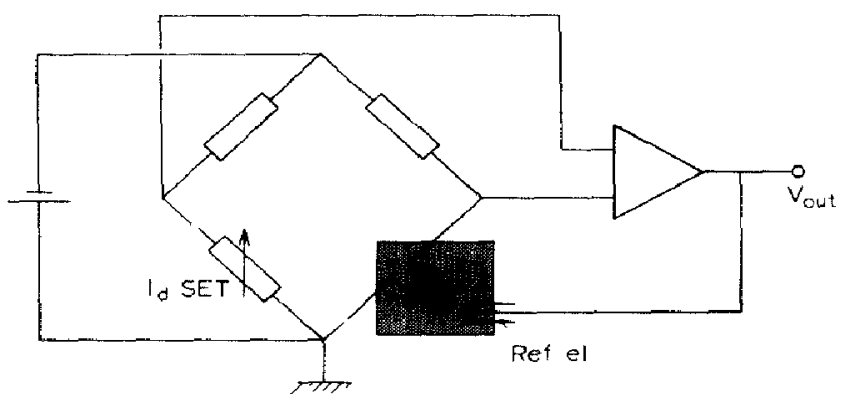

Fig 5 Principle diagram of an automatically balanced bridge circuit

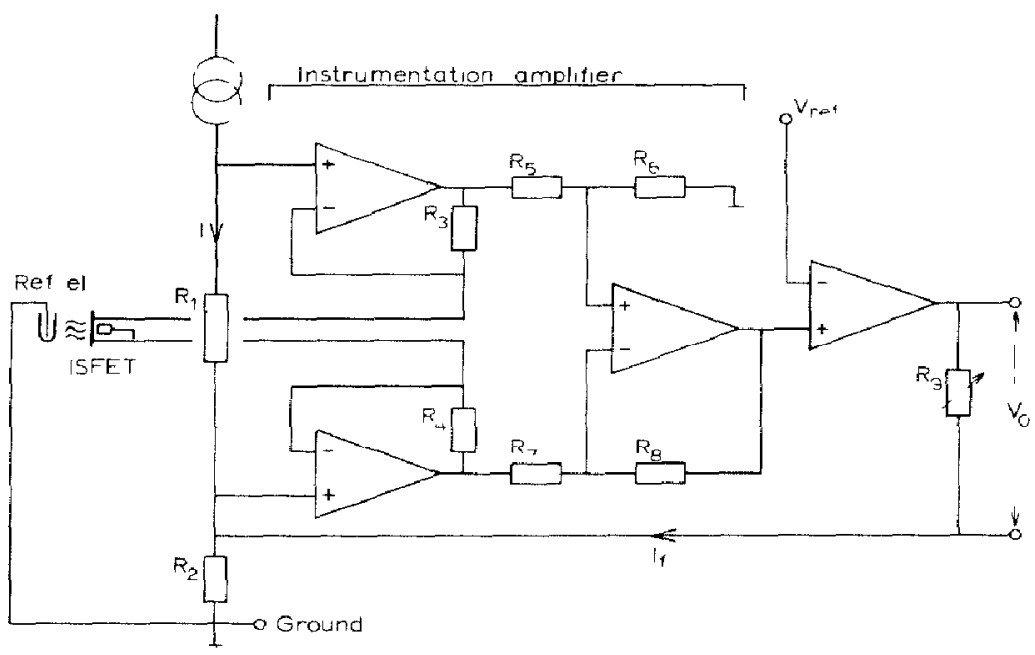

Fig 6 Principle diagram of a source and drain follower

instrumentation amplifier is now inversely proportional to the channel resistance of the ISFET Note that the connections for source and drain are of very low resistance, which means that the ISFET can be connected to the system by means of long, unshielded wures, insensitive to interference from external electric fields and static charges

The difference between the output voltage of the instrumentation amplifier and an adjustable reference voltage, $V_{\text {ref }}$, is amplified by the operational amplifier, from which the output 'injects' a feedback current $I_{\mathrm{f}}$ into $R_{2}$, thus controlling the source and drain voltages which are equal to $V_{\mathbf{R}_{2}}$ and $V_{\left(\mathrm{R}_{2}+\mathrm{R}_{1}\right)}$, respectively This control action results in a constant $I_{\mathrm{d}}$ at a constant $V_{\mathrm{d}}=I \mathrm{R}_{1}$ or, in other words, $\imath_{\mathrm{d}}=0, v_{\mathrm{d}}=0$, while the feedback causes $v_{\mathrm{g}}=0$ The feedback current is measured $v$ sa the adjustable resistor $R_{9}$ If the open-loop amplification of the system, determined by amplification of the combination of ISFET and instrumentation amplifier (approximately $R 8 / R 7 \times S \times\left(R_{3}+R_{4}\right)$, and the open-loop amplification of the operational amplifier is high enough, the source and drain potential with regard to ground follow a change in the effective input voltage $v_{1}$ of the 
ISFET At the same time, the amphified input voltage is avalable across $R_{9}$ according to

$$
v_{0}=\frac{\mathbf{R}_{9}}{\mathbf{R}_{2}} v_{1}
$$

Note that, due to the fact that the potential of the source and drain leads follows the input potential with regard to earth, no capacitive loads exist, which gives the system a maximum of frequency response, independent of the length of the connecting leads Further, the system includes a very simple calibration possibility, which corresponds to the usual calibration facilities of $\mathrm{pH}$ measurements, namely, the starting point that $\mathrm{pH}=7$ corresponds to a voltage of $0 \mathrm{~V}$

The procedure of a $\mathrm{pH}$ measurement with this system is as follows If the ISFET is placed in a buffer of $\mathrm{pH}=7$, the reference voltage is adjusted in such a way that $I_{f}=0$ The output voltage across $R_{9}$ is thus also zero, independent of the value of $R_{9}$ If the ISFET is then placed in another buffer, $e g, \mathrm{pH}=4$, the value of $\mathrm{R}_{9}$ can be adjusted in such a way that an appropriate voltage is measured, $e g, 3 \mathrm{~V}$ if the desired sensitivity of the system should be $1 \mathrm{~V} / \mathrm{pH}$ of course the output voltage can be directly calibrated in $\mathrm{pH}$ units

As already mentioned in another paper [8], the reference point $\mathrm{pH}=7$, corresponding to $V_{0}=0 \mathrm{~V}$, should be independent of temperature to facllitate absolute measurement With the development of glass membrane electrodes, this problem is solved in a technological way by the choice of the inner buffer solution The question arises whether a similar technological solution can also be found for an ISFET Therefore, we have to focus our attention on the basic equation of the ISFET and determine it in view of the temperature sensitivity of all terms

\section{The temperature sensitivity of ISFETs} by [8]

The equation for the $\mathrm{d} c$ current, $I_{\mathrm{d}}$, of a $\mathrm{pH}$-sensitive ISFET is given

$$
\begin{gathered}
I_{\mathrm{d}}=\beta\left[\left(V_{\mathrm{g}}-E_{\mathrm{ref}}+\Delta \phi_{\mathrm{j}}+\phi_{\mathrm{o}}+\frac{R T}{F} \ln a_{\mathrm{H}^{+}}+\Phi_{\mathrm{S}_{\mathrm{l}}}+\frac{Q_{\mathrm{ss}}+Q_{\mathrm{ox}}+Q_{\mathrm{B}}}{C_{\mathrm{ox}}}-\right.\right. \\
\left.\left.-2 \Phi_{f}\right) V_{\mathrm{d}}-\frac{1}{2} V_{\mathrm{d}}^{2}\right]
\end{gathered}
$$

where $\beta=\mu C_{\mathrm{ox}} W / L$ is a geometric constant

$V_{\mathrm{g}}$ is the potential applied to the reference electrode with regard to the source,

$E_{\text {ref }}-\Delta \phi$, is the voltage of the reference electrode including the liquid junction potential,

$\phi_{0}+\frac{R T}{F} \ln a_{\mathrm{H}^{+}}$is the voltage across the electrolyte-oxide interface, 
$\Phi_{S_{1}}$ is the slicon work function,

$Q_{s s}$ is the charge of the interface states,

$Q_{o x}$ is the charge in the oxide,

$Q_{\mathrm{B}}$ is the depletion charge in the bulk,

$\Phi_{f}$ is the bulk Fermı potential,

$V_{\mathrm{d}}$ is the $\mathrm{d} c$ drain to source voltage,

$C_{\text {ox }}$ is the oxide capacity per unit area

In the electronic circuit as given in Fig $6, V_{\mathrm{d}}$ is kept constant, while $I_{\mathrm{d}}$ can be set to a desired value, which is also kept constant due to the feedback For $\mathrm{pH}=7$ this value is chosen in such a way that the feedback current $I_{\mathrm{f}}$ is zero $\left(V_{\mathrm{o}}=0\right)$, resulting in a certain value of $V_{\mathrm{g}}$ which can be derived from eqn (12)

$$
\begin{gathered}
V_{\mathrm{g}}=\frac{\mathrm{const}}{\beta}+E_{\mathrm{ref}}-\Delta \phi_{\mathrm{j}}-\phi_{\mathrm{o}}-\frac{R T}{F} \ln a_{\mathrm{H}^{+}}-\Phi_{\mathrm{S} 1} \\
-\frac{Q_{\mathrm{ss}}+Q_{\mathrm{ox}}+Q_{\mathrm{B}}}{C_{\mathrm{ox}}}+ \\
+2 \Phi_{\mathrm{f}}
\end{gathered}
$$

For $\mathrm{pH}=7$ it ylelds

$$
\frac{\mathrm{d}}{\mathrm{d} T}\left(-\frac{R T}{F} \ln a_{\mathrm{H}^{+}}\right)=139 \mathrm{mV} /{ }^{\circ} \mathrm{C},
$$

which means that by maintaining $I_{\mathrm{f}}=0$, independent of temperature, the condition given in the next equation should be fulfilled

$$
\begin{aligned}
\frac{\mathrm{d}}{\mathrm{d} T}\left(\frac{\text { const. }}{\beta}+E_{\mathrm{ref}}-\Delta \phi_{\mathrm{I}}-\phi_{\mathrm{o}}-\Phi_{\mathrm{S} 1}\right. & \left.-\frac{\left(Q_{\mathrm{ss}}+Q_{\mathrm{ox}}+Q_{\mathrm{B}}\right)}{C_{\mathrm{ox}}}+2 \Phi_{\mathrm{f}}\right)= \\
& =-139 \mathrm{mV} /{ }^{\circ} \mathrm{C}
\end{aligned}
$$

Of course the same equation has to be obeyed if the bridge circuit as given in Fig 5 is used

The requirement given in eqn (15) differs from the corresponding one for glass membrane electrodes [8], in the first place due to the fact that now solid-state parameters are part of the equation Further, the reference electrode voltage and the standard potential of the electrolyte-oxide interface are now also part of the equation

It is unrealistic to assume that the ISFET process technology should be so accurate that the requirement as given in eqn (15) can be met by a technological process control as is the case for glass membrane electrodes [8] Fortunately, we have, in contrast to glass membrane electrodes, electronic possibilities to tackle the problem, which will be further discussed in this Section

In general, it can be stated that a compensation for temperature drift, which requires adjustment for each individual ISFET, is not convenient with regard to the desired interchangeablity of the devices A necessary calibration for the input varnable, $e g$, the $\mathrm{pH}$, may already be less desurable, this cannot in any way be accepted with regard to an interference signal, as is the temperature in this case 
A usual approach in electronics to compensate for temperature drift in solid-state devices is to create a differential pair on one chip from which one device is the active input device and the other is used for temperature compensation, assuming that the temperature characteristics of both devices are equal As can be seen from Fig 7, which shows a generalized $I_{\mathrm{d}}-V_{\mathrm{g}}$ curve of a MOSFET or an ISFET as a function of temperature, the requirement $\mathrm{d} I_{\mathrm{d}_{1}} / \mathrm{d} T=\mathrm{d} I_{\mathrm{d}_{2}} / \mathrm{d} T$ for a pair of devices having the same characteristics, can only be maintained if, in addition, the electrical bias of both devices is kept equal $\left(I_{\mathrm{d}_{1}}=I_{\mathrm{d}_{2}}\right.$ and $\left.V_{\mathrm{g}_{1}}=V_{\mathrm{g}_{2}}\right)$

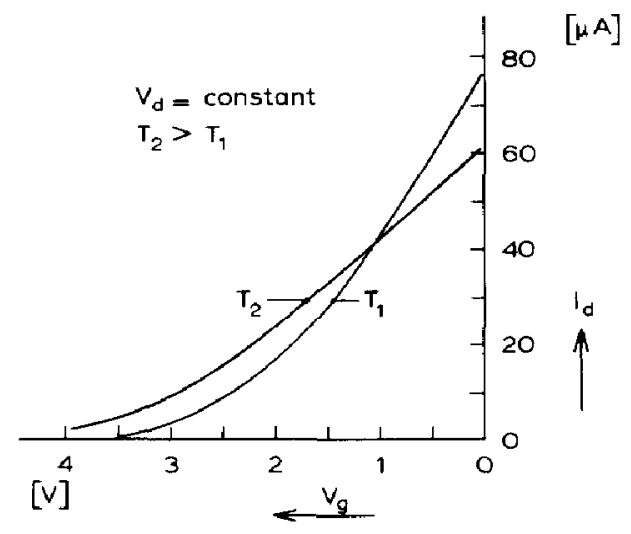

Fig 7 Generalized $I_{\mathrm{d}}-V_{\mathrm{g}}$ curve of a MOSFET or ISFET as function of temperature

Both requirements can be met reasonably for a pair of MOSFETs with today's MOSFET technology and the application of electronic feedback (see Fig 8(a)) It 1s, however, not realistic to use this approach for a pair consisting of an ISFET and a MOSFET on the same chip [9], due to the explicit existence of differences in $V_{t}$, resulting in a bias difference (see Fig. 8(b)) This is the reason why this system needs adjustment for each individual probe, calıbrated by means of a known temperature variation, as reported by McKinley [10] A principally better approach to solve the problem of automatic compensation for temperature interference is the construction of a differential ISFET pair, one for the measurement of the $\mathrm{pH}$ and one with a

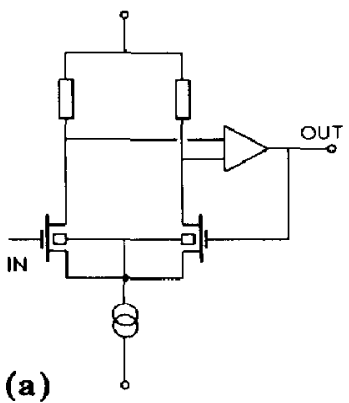

(a)

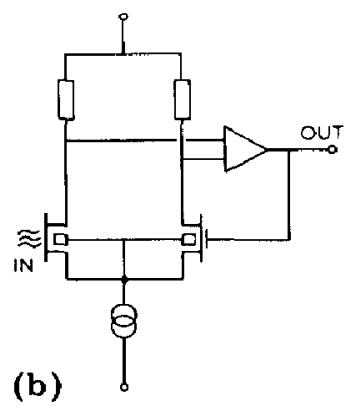

(c)

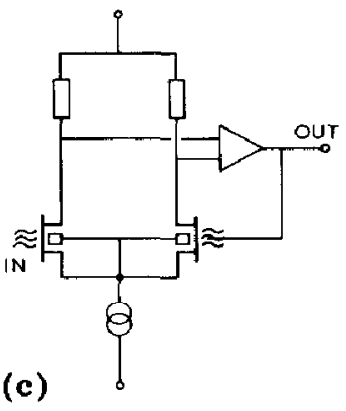

Fig 8 Principle diagrams for differential amplifier circuit with feedback for (a) a pair of MOSFETs, (b) an ISFET and a MOSFET, (c) a pair of ISFETs 
separate compartment on top of the gate, filled with a buffered agarose, which is in contact with the solution to be measured via a liquid junction, as described by Janata and Huber [11] and shown in Fig 9

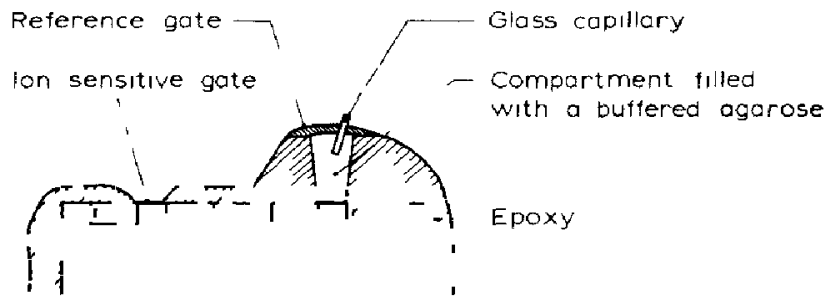

Fig 9 Construction of differential pair of a pH ISFET and a reference ISFET (after Janata and Huber [10])

A practical problem with this construction is that the reference ISFET cannot be made completely by a technology which is compatible with ICtechnology Further, the construction introduces a difference in electrical bias due to the additional liquid junction potential for the reference ISFET This will, of course, also be the case for a difference between the $\mathrm{pH}$ of the solution to be measured and the buffer solution of the reference ISFET, if this is not compensated by a feedback system Then, however, the possibility must be present to contact the liquid gate of the reference ISFET (the buffer solution) separately (see Fig 8(c)) Therefore, this system has to be further investigated electronically as well as technologically

The conclusion is that the approach of a differential pair construction on one chip to prevent temperature drift, as commonly in use for MOSFETs, cannot be applied directly to ISFETs

An unusual approach in conventional electronics is the simultaneous detection of those parameters of the measuring device which are responsible for the temperature dnft, and to use this signal for compensation

The function $I_{\mathrm{d}}(T)$ or, in the feedback circuit of Fig 6 , the corresponding function $V_{\mathrm{g}}(T)$ (multiplied by a constant factor) is unknown, in such a way that the theory concerning the temperature sensitivity of the terms $\beta$ and $\Phi_{f}$ contains some empirical coefficients

$$
\begin{aligned}
& \beta=C_{\mathrm{ox}} \frac{W}{I} T^{a}-1<a<-15 \\
& \Phi_{\mathrm{f}}=\frac{k T}{q} \ln \frac{N_{\mathrm{A}}}{C T^{-3 / 2} \exp \left(-W_{\mathrm{g}} \mathrm{S}_{1} / T\right)}
\end{aligned}
$$

Therefore, the simultaneous measurement of the temperature with a separate sensor cannot be used for compensation of temperature drift in $V_{\mathrm{g}}(T)$ for $I_{\mathrm{d}}=$ constant Instead of this, we have continuously to measure the unknown function for each individual ISFET connected to the amplifier during operation With this measure, the set value of $I_{\mathrm{d}}$ (see Fig 6) can be controlled in such a way that $V_{\mathrm{g}}=$ constant The same signal can be used to 
adjust the amplification of the measured output signal as a function of $\mathrm{pH}$, in agreement with the slope correction of glass membrane electrodes

It has already been shown that for MOSFETs the substrate or bulk, which up to now in this paper has been assumed to be shortened with the source, can be used as an additional signal input In this case we have to extend eqn (1) with terms which reflect the influence of the bulk to source voltage $V_{\mathrm{b}}$, resulting in eqn (16)

$$
I_{\mathrm{d}}=\beta\left[\left(V_{\mathrm{g}}-V_{\mathrm{t}}\right) V_{\mathrm{d}}-\frac{1}{2} V_{\mathrm{d}}^{2}-\frac{2}{3} \alpha\left\{\left(V_{\mathrm{d}}-V_{\mathrm{b}}+2 \Phi_{\mathrm{f}}\right)^{3 / 2}-\left(V_{\mathrm{b}}+2 \Phi_{\mathrm{f}}\right)^{3 / 2}\right\}\right]
$$

where $\alpha=\left(2 \epsilon_{0} \epsilon_{\mathrm{S} 1} q N_{\mathrm{A}}\right)^{1 / 2} / C_{\mathrm{ox}}$ and the further symbols being already mentioned in the preceding text

For the small-signal behaviour of the bulk, differentiation of eqn (16) gives the mutual conductance of the bulk

$$
S_{\mathrm{b}}=\left.\frac{\mathrm{d} I_{\mathrm{d}}}{\mathrm{d} V_{\mathrm{b}}}\right|_{V_{\mathrm{d}}=\text { const }}=\alpha \beta\left[\left(V_{\mathrm{d}}-V_{\mathrm{b}}+2 \Phi_{\mathrm{f}}\right)^{1 / 2}-\left(-V_{\mathrm{b}}+2 \Phi_{\mathrm{f}}\right)^{1 / 2}\right]
$$

As can be seen from eqns (16) and (17), $S_{\mathrm{b}}$ and $I_{\mathrm{d}}$ contain the same temperature dependent terms, $\beta$ and $\Phi_{\mathrm{f}}$ It can be shown [7] that $\Delta I_{\mathrm{d}} / \Delta S_{\mathrm{b}}$ is a constant independent of $V_{\mathrm{g}}$, which means that a simultaneous measurement of $\Delta S_{\mathrm{b}}(T)$ reflects the temperature sensitivity of $I_{\mathrm{d}}\left(\Delta I_{\mathrm{d}}(T)\right)$ This means for an ISFET that, so far as it concerns the temperature sensitive solid-state parameters, an additional signal can be withdrawn from the device, $\Delta S_{\mathrm{b}}(T)$, independent of the input signal $(\mathrm{pH})$, which can be used for readjustment of $I_{\mathrm{d}} v i a$ the reference potential $V_{\text {ref }}$ in the circuit of Fig 6 during operation The result will be that the ISFET, adjusted in a certain bias condition for the reference point $\mathrm{pH}=7$, will maintain this condition automatically

The realisation of this system is quite simple as is shown in Fig 10 In order to create a sensitivity independent of $\beta, R_{\mathrm{s}}$, and $R_{\mathrm{d}}$, the feedback system of Fig 6 is used In this system, an additional sinusoidal signal is injected by means of a transformer that is connected between the source and the bulk

Because the whole feedback system is limited to a frequency of $3 \mathrm{kHz}$, the frequency of the bulk signal is chosen as $30 \mathrm{kHz}$, and is thus not affected by the feedback loop The amplitude of the $30 \mathrm{kHz}$ signal is measured at the output of the instrumentation amplifier by means of a lockin amplifier, and appears to be proportional to $\Delta S_{\mathrm{b}}$ This signal is used to readjust the set reference voltage $V_{\text {ref }}$

Considering eqn (15), it will be obvious that the temperature compensation mentioned above only yields for the terms const $/ \beta(T)$ and $\Phi_{\mathrm{f}}(T)$ does not correct changes in the voltage of the reference electrode $\left(E_{\mathrm{ref}}-\Delta \phi_{1}\right)$ and the electrolyte-oxide standard potential, $\phi_{0}$, as a function of the temperature If an external reference electrode is used it is clear that this reference electrode has to be kept at a constant temperature It remains to be seen whether an over compensation of the terms const $/ \beta(T)$ and $\Phi_{f}(T)$ 


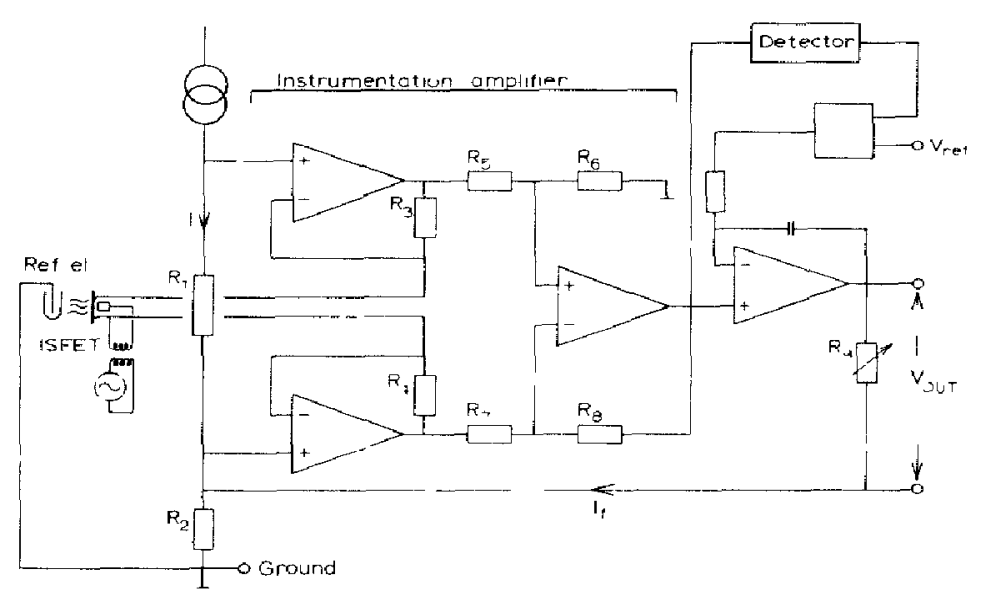

Fig 10 Principle diagram of an ISFET amplifier with additional circuitry for temperature measurement and compensation

may also include the effect of $\phi_{0}(T)$ This may possibly also yield for a reference electrode integrated with the ISFET, and thus subject to the same temperature variations In this respect, eqn (15) can possibly be obeyed As the equation does not give a direct indication for the best value of over compensation, this has to be determined empirically

\section{Conclusion}

It can be concluded that ISFETs can be seen as a new class of ion sens1tive devices which greatly differs from the conventional potentiometric $\mathrm{pH}$ sensor in the sense of being an electronic component which can be controlled by electronic means such as feedback, simultaneous parameter measurement with corresponding compensation, etc On the other hand the ISFET differs from the original electronic component, the MOSFET from which the ISFET is denved, in the sense of having additional parameters of non-physical ongin, which cannot be directly influenced by electronic means It is useful to investigate whether a total feedback system including the actual pH unit may solve this problem Such an approach requires, however, the development of an electronic $\mathrm{pH}$ actuator This development would lead to far-reaching progress in the application of the devices

\section{References}

1 M Esashi and $T$ Matsuo, Integrated micro mult 1 ion sensor using field effect of semiconductor, IEEE Trans Blomed Eng, 25 (1978) 184 - 191

2 S D Moss et al, pH Catheter tip semiconductor micro electrode, in Theory, Design and Blomedical Applications of Solld State Chemical Sensors, CRC Press, 1978, p 124

3 P Bergveld and N F de Rooly, The single electrode operation of an ion-sensitive field 
effect transistor, in Theory, Destgn and Biomedical Applications of Solid State Chemical Sensors, CRC Press, 1978, p 148

4 H E Cline and T B Anthony, High-speed droplet migration in silicon, $J$ Appl Phys , 47 (1976) 2325 - 2331

$5 \mathrm{C} C$ Wen et al, Gate-controlled diodes for ionic concentration measurement, $I E E E$ Trans Electron Devices, 26 (12) (1979) 1945 - 1951

6 J Janata, Thermodynamics of chemically sensitive field effect transistors, in Theory, Design and Biomedical Applications of Solld State Chemical Sensors, CRC Press, 1978 , p 48

7 P Bergveld, Electronic circuit design principles for parameter control of ISFETs and related devices, Med Biol Eng, 17 (1978) 655-661

8 P Bergveld and N F de Roov, The history of chemically sensitive semiconductor devices, Sensors and Actuators, 1 (1981) 5 - 15

$9 \mathrm{P} W$ Cheung et al, Theory, fabrication, testing and chemical response of ion sensitive field effect transistor devices, in Theory, Design and Btomedical Applications of Solvd State Chemical Sensors, CRC Press, 1978, p 98

10 B A McKinley et al, In vivo continuous monitoring of $\mathrm{K}^{+}$in animals using ISFET probes, Med Instrum (Baltımore), 14 (2) (1980) $93-97$

$11 \mathrm{~J}$ Janata and $\mathrm{R} J$ Huber, Ion sensitive field effect transistors, Ion Select Electrode Rev, 1 (1979) $31-79$

12 Leistiko et al, Electron and hole mobility in inversion layers and thermally oxidized silicon surfaces, IEEE Trans Electron Devices, 12 (1965) 248 - 254

13 L Vadasz and A S Grove, Temperature dependence of MOS transistor characteristics below saturation, IEEE Trans Electron Devices, 13 (1966) 863 - 866 